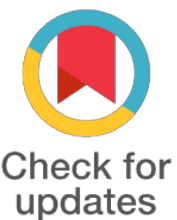

updates
*For correspondence:

vbngoc@hcmus.edu.vn

Competing interests: The authors declare that no competing interests exist.

Received: 2017-08-10

Accepted: 2017-08-19

Published: 2017-09-05

Copyright The Author(s) 2017. This article is published with open access by BioMedPress (BMP).

This article is distributed under the terms of the Creative Commons Attribution License (CC-BY 4.0) which permits any use, distribution, and reproduction in any medium, provided the original author(s) and the source are credited.

\section{Establishing an artificial cartilage from rabbit bone marrow derived mesenchymal stem cell and polycaprolactone scaffold}

\author{
Thuy Thi-Thanh Dao, Liem Hieu Phan, Ha Thi-Ngan Le, Lan Thi Phi, Phuc Van \\ Pham, Ngoc Bich Vu
}

Laboratory of Stem Cell Research and Application, University of Science, Vietnam National University HCMC, Ho Chi Minh City, Vietnam

\section{Abstract}

Nowaday, the regenerative medicine based on the use of biomaterial scaffold combine to stem cells was considered as a necessary treatment strategy for injury or diseases involving cartilage. In this study, we investigated the adherence, proliferation and chondrogenic differentiation of rabbit bone marrow-derived mesenchymal stem cells (rBM-MSCs) on biodegradable polycaprolactone scaffold (PCL). MSCs were isolated from bone marrow of rabbit. rBM-MSC (2x105cell/scaffold) were seeded on PCL and collagen-coated PCL (PCL/col) scaffolds. The cell

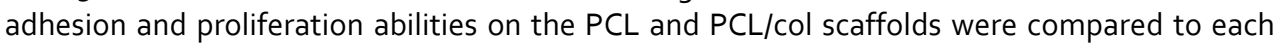
other to find the better using MTT assay. Then $3 \mathrm{D}$ cultured-cells were induced in chondrogenic differentiation medium. The change of scaffold surface structure was evaluated by scanning electron microscope (SEM). The accumulation of aggrecan protein was detected by Alcian Blue staining. The chondrogenic-related gene expression such as sox9, collagen type 1 , collagen type 2, collagen type 10, aggrecan, runx2 was assessed by RT-PCR. The existence of artificial cartilage after transplantation of chondrocyte-seeded scaffold under rabbit skin was evaluated by staining histological sections with Alcian Blue. The results showed that rBM-MSCs attached and grown on PCL/col and PCL scaffolds. However, the adhesion efficient of rBM-MSC on $\mathrm{PCL} / \mathrm{col}$ scaffold was better than PCL scaffolds. Therefore, $\mathrm{PCL} / \mathrm{col}$ scaffold was used for next experiments. The morphology of rBM-MSCs on PLC/col scaffold were changed and the aggrecan accumulation increased after cultured in chondrogenic differentiation medium. The scaffold surface modification was detected with the presentation of collagen-like fiber structures. The expression of chondrogenesis-associated genes increased after 3, 7, 21, 28 days of chondrogenic induction, especially for collagen type II, collagen type I, aggrecan and collagen type X. Transplanted artificial cartilage was existed in rabbit for six weeks. The mature chondrocyte-like cells were detected and cartilage specific-proteoglycan accumulated more in differentiated cell-scaffold than scaffold seeded undifferentiated-cells. Thus artificial cartilage could be created when differentiating rBM-MSC into chondrocyte on collagen-coated PCL scaffold.

\section{Keywords}

3D scaffold, artificial cartilage, biodegradable scaffold, bone marrow, chondrocyte, mesenchymal stem cell, polycaprolacton 


\section{Funding}

VNU-HCM under grant number C2017-18-24/HĐ-KHCN

\section{References}

1. Adelola O. Oseni, C.C., Maria Z. Boland, Peter E. Butler and Alexander M. Seifalian, Cartilage Tissue Engineering: the Application of Nanomaterials and Stem Cell Technology. Tissue Engineering and Regenerative Medicine, Tissue Engineering for Tissue and Organ Regeneration, 2011.

2. Dawson, E., et al., Biomaterials for stem cell differentiation. Adv Drug Deliv Rev, 2008. 6o(2): p.

215-28.

3. Elisseeff, J., et al., The role of biomaterials in stem cell differentiation: applications in the musculoskeletal system. Stem Cells Dev, 2006. 15(3): p. 295-303. 4. Ha Thi Ngan Le, Lan Thi Phi, Thuy Thi Thanh Dao, Ngoc Kim Phan, Phuc Van Pham, Ngoc Bich $\mathrm{Vu}$, A mouse model of osteonecrotic femoral head induced by methylprednisolone and liposaccharide, Biomedical Research and 2016. 5. Hwang, N.S., et al., Regulation of osteogenic and chondrogenic differentiation of mesenchymal stem cells in PEG-ECM hydrogels. Cell Tissue Res, $2011.344(3)$ : p. 499-509. 6. Portocarrero G, C.G.a.L.A.T., Challenges in Cartilage Tissue Engineering. Tissue Science \& Engineering, 2013. 